\title{
CONSTRUCCIÓN DE UN INVENTARIO DE INTEGRACIÓN FAMILIAR
}

\section{Construction of a Family Integration Inventory}

\begin{abstract}
Walter Arias Gallegos*, Rodolfo Castro Salinas**, Sergio Dominguez Lara***, María Alejandra Masías Salinas****, Fiorela Canales Rivera*****, Sylvana Castilla Querevalü******, Selene Castilla Querevalü*******
\end{abstract}

\begin{abstract}
Resumen
El presente artículo describe el proceso de construcción de un Inventario de Integración Familiar que consta de 52 ítems dispuestos en una escala tipo Likert, y cuenta con criterios de validez y confiabilidad óptimos $(\alpha=.739)$. Se obtuvo un valor KMO de 922 que admite la factibilidad de realizar un análisis factorial exploratorio. Como producto del análisis factorial en dos etapas se obtuvo por resultado un instrumento unidimensional. Asimismo, se establecieron los baremos con los respectivos percentiles para los valores obtenidos: alto, medio y bajo.
\end{abstract}

Palabras clave: Integración familiar, validez, confiabilidad.

\begin{abstract}
The present article describes the process of construction of a Family Integration Inventory which contents 52 items distributed in a Likert scale, and has optimum criteria of validity and reliability $(\alpha=.739)$. We obtain a KMO value of .922 which admits the factorization of the instrument As result of the factorial analysis applied in two moments we finally have a unidimensional inventory. Moreover, we settle the percentiles in three levels of family integration: high, medium and low.
\end{abstract}

Key words: Family integration, validity, reliability.

\footnotetext{
* Profesor de la Universidad Católica San Pablo y director del Grupo de Investigación Psyché. E-mail: walterlizandro@hotmail.com

** Profesor e investigador de la Universidad Católica San Pablo.

*** Profesor de la Facultad de Psicología y Trabajo Social de la Universidad Inca Garcilaso de la Vega.

**** Estudiante de psicología de la Universidad Católica San Pablo y coordinadora del Grupo de Investigación Psyché.

***** Estudiante de psicología de la Universidad Católica San Pablo y miembro del Grupo de Investigación Psyché.

******* Estudiante de psicología de la Universidad Católica San Pablo.

******* Estudiante de psicología de la Universidad Católica San Pablo.
} 


\section{INTRODUCCIÓN}

El estudio de la familia, ha sido ciertamente recurrente en nuestro país. Algunos de estos estudios han estado destinados a reflexionar sobre el impacto de la familia sobre la educación (Pinzas, 1987; Jadue, 1996, 2000; Baessa y Fernández, 2003), mientras que otros se han ocupado de evaluar la satisfacción familiar (Sobrino, 2008; Tirado, Álvarez, Chávez, Holguín, Honorio, Moreno, Sánchez, Shimajuko y Uribe, 2008, Mayorga y Ñiquen, 2010), medir la satisfacción marital (Rebaza y Julca, 2009; Prado y Del Águila, 2010; Eguiliuz, Calvo y de la Orta, 2012), valorar los efectos de la estructura familiar en diversas variables psicológicas (Prado y Del Águila, 2004; Oporto y Zanabria, 2006; Silva \& Argote, 2007; Laguna \& Rodríguez, 2008) y profundizar en los vínculos afectivos entre los padres y los hijos (Portilla, Salinas y Bueno, 2006; Portilla, Romero y Gonzales, 2008; Yaya, Vilches y Portilla, 2009). No obstante, han sido escasos los estudios que han utilizado instrumentos para investigar la familia elaborados en el país, antes bien, se han estandarizado y validado diversas pruebas para estos fines (Merino, Díaz y DeRoma, 2004).

En ese sentido, en este artículo, mostramos el proceso de construcción de una prueba psicométrica que mide la integración familiar, que ha sido validada en la ciudad de Arequipa. Hemos definido la integración familiar como el grado de salud, equilibrio y armonía de las relaciones que nacen del vínculo conyugal y que naturalmente se orienta a satisfacer la necesidad de trascendencia personal en base al respeto, el diálogo y la comunión entre sus miembros considerando sus responsabilidades, según el ciclo vital de la familia. Partimos del hecho -en base a diagnósticos socialesque la familia se encuentra en crisis, constatamos que existen muchas familias fragmentadas y divididas, y que en esa medida, sería de gran utilidad medir el grado de integración de las familias.

Las causas de la desintegración familiar son muchas, todas ellas se ubican en el crisol de la sociedad, ya que en las familias se manifiestan las condiciones sociales, culturales y económicas de la época. Así pues, la pobreza, la falta de educación y madurez de los padres que forman una familia, son relevantes para explicar las condiciones presentes de las familias. En ese sentido, las familias han cambiado drásticamente en los últimos 50 años. Estos cambios se manifiestan en la estructura familiar, el tamaño de las familias, la preparación académica de sus miembros y los valores familiares (Covarrubias y Gómez, 2012). Con respecto a la estructura familiar, de los modelos nucleares tradicionales, se ha pasado a modelos familiares monoparentales o familias reconstruidas, y diversas tipologías de familia que distorsionan su naturaleza más íntima, como las uniones libres de personas del mismo sexo (Guerra, 2004).

El tamaño familiar también ha cambiado, ya que de tener familias numerosas se ha pasado a tener familias con limitada prole. El número de hijos promedio de la familia peruana es de 2.1 (INEI, 2013), mientras que en el mundo la media es apenas de 2.55. En la Tabla 1 se puede apreciar las tasas de fecundidad de la mujer desde 1955 hasta el 2010, según los diversos continentes. Es evidente el paulatino descenso del número de hijos por mujer, las repercusiones económicas y sociales de las familias poco numerosas, han sido perjudiciales para las naciones. Por tal motivo, en los últimos años, los países de estas regiones han intentado revertir esta situación.

Tabla 1.

Evolución de tasas de fecundidad en América Latina y otras regiones

\begin{tabular}{lcccccc}
\hline Región & $\mathbf{1 9 5 5 -}$ & $\mathbf{1 9 6 5 -}$ & $\mathbf{1 9 7 5 -}$ & $\mathbf{1 9 8 5 -}$ & $\mathbf{1 9 9 5 -}$ & $\mathbf{2 0 0 5 -}$ \\
& $\mathbf{1 9 6 0}$ & $\mathbf{1 9 7 0}$ & $\mathbf{1 9 8 0}$ & $\mathbf{1 9 9 0}$ & $\mathbf{2 0 0 0}$ & $\mathbf{2 0 1 0}$ \\
\hline Mundo & 4.96 & 4.90 & 3.92 & 3.38 & 2.80 & 2.55 \\
Regiones & & & & & & \\
más desarrolladas & 2.82 & 2.37 & 1.91 & 1.83 & 1.55 & 1.60 \\
África & 6.82 & 6.80 & 6.61 & 6.13 & 5.28 & 4.67 \\
Asia & 5.63 & 5.67 & 4.19 & 3.40 & 2.67 & 2.34 \\
Europa & 2.66 & 2.36 & 1.97 & 1.83 & 1.40 & 1.45 \\
América Latina & 5.94 & 5.57 & 4.50 & 3.42 & 2.74 & 2.37 \\
Oceanía & 4.09 & 3.57 & 2.73 & 2.51 & 2.42 & 2.30 \\
\hline
\end{tabular}

Fuente: Tomado de Arellano, R. (2012)

En ese sentido el costo social de limitar los hijos a través de políticas de control de la natalidad, implicó una inversión de la pirámide poblacional que hizo sentir sus efectos en áreas como la salud (a mayor número de personas senectas mayores tasas de enfermedad), economía (menor número de gente joven trabajando y mayor el número de gente jubilada) y la sociedad 
(países sin medidas adecuadas para brindar bienestar social a gente mayor). Por otro lado, la preparación académica ha aumentado, y aunque esta debiera ser una fortaleza de la familia, en muchos casos ha sido instrumento para difundir ideas distorsionadas de la sexualidad, el matrimonio y la paternidad (Rodríguez, 2006). Los valores familiares también han cambiado, en la medida que se han perdido espacios de comunicación familiar (Sobrino, 2008), y valores como la obediencia y el respeto a los padres como figuras de autoridad y amor (Rodríguez, 2008).

La familia por tanto debe enfrentar estas cuestiones como desafíos serios, ello supone desde un ángulo académico, profundizar más en el estudio de las implicancias de las nuevas estructuras familiares y cómo se ordenan sus miembros en torno a los nuevos valores familiares (Perriaux, 2011), más aún si la familia es un vehículo para el bienestar, la salud y el desarrollo social. Los estudios en nuestro país, por ejemplo, señalan que la satisfacción familiar entendida como el conjunto de sentimientos positivos que aparecen en la persona a partir de la relación de convivencia con los miembros que conforman el sistema familiar-, se relaciona negativamente con desórdenes mentales como ansiedad, trastornos del sueño y depresión (Tirado et al., 2008). Asimismo, se ha probado la importancia del rol de los padres en el éxito escolar de los hijos (Jadue, 1996, 2000; Baessa y Fernández, 2003), y la trascendencia de los vínculos afectivos entre los padres e hijos, en su futuro desarrollo (Portilla, Salinas y Bueno, 2006; Portilla, Romero y Gonzales, 2008). De hecho los procesos emocionales de los padres tienen impacto en la crianza de los hijos (Merino, Díaz y DeRoma, 2004), que pueden devenir en la aparición de trastornos conductuales (Arias, 2013).

En suma, la familia es importante, porque en ella se fragua el futuro de la humanidad (Juan Pablo II, 1981), de ahí que la integración familiar es una característica fundamental en las familias, ya que en la medida que todos y cada uno de los integrantes del sistema familiar se van ajustando psicológica y socialmente entre sí, los conflictos familiares van disminuyendo, generando mayor bienestar personal y familiar (Sobrino, 2008). Sin embargo, la integración familiar puede verse afectada por factores propios del ciclo natural de desarrollo de la familia, como por eventos inesperados que debilitan el ajuste de sus miembros (Ríos, 2003). Cabe tener siempre presente, que en tanto la familia sea un sistema organizado, y el factor formador de dicho sistema lo constituyen los lazos de consanguineidad (Hellinger, 2002, 2003, 2005), deben haber límites claros y roles establecidos entre los miembros de la familia, en función del ciclo vital en que se encuentre dicho sistema (Minuchin y Fishman, 1996; Haley, 2002).

Estos vínculos de consanguineidad devienen de la unión estable entre varón y mujer que procrean por amor (Scola, 2001) y conviven en constante comunión de intereses y valores (Cafarra, 2011) que determinan la calidad de las relaciones de sus miembros y el establecimiento de metas comunes (Serreti, 2011). En ese sentido, el soporte de la familia viene dado por la relación de los padres -en tanto pareja y como padres propiamente (Arias, 2011)-, que también está inmersa en el ciclo evolutivo de la familia (Rebaza y Julca, 2009), y que resulta determinante en la manera cómo sus miembros se comunican, se integran y se adaptan a su entorno, porque los padres modelizan diversos comportamientos de sus hijos (Salvatierra, 1992, 1993, 1997).

En ese contexto nos planteamos un doble objetivo, por un lado construir y validar una prueba psicométrica que mida la integración familiar, y por otro, valorar los niveles de integración familiar en las familias de Arequipa. En este trabajo solo presentaremos el proceso de construcción del instrumento, ya que los resultados de la evaluación se presentarán por separado en otro informe.

\section{MÉTODO}

Para la construcción del material se trabajó dentro del marco de la teoría del análisis factorial de los ítems, y se procedió según criterios psicométricos que aseguren la validez y confiabilidad del mismo. Ello supone que nuestro estudio es de tipo básico y cuantitativo. Como marco epistemológico del instrumento se tiene el realismo filosófico y el enfoque familiar sistémico.

\section{Participantes}

La muestra está constituida por 334 personas de 
trece distritos de la ciudad de Arequipa que se ubican en la zona Metropolitana. La composición de la muestra por distrito se aprecia en la Tabla 2. Como criterios de inclusión, se han tenido en cuenta que los encuestados: sean padres o madres de familia, jefes de hogar, ya sea que cohabiten con sus parejas o no, o que se hayan unido en matrimonio o que solamente convivan.

\section{Tabla 2}

\section{Composición de la muestra por distritos}

\begin{tabular}{lc}
\hline Distrito & Muestra \\
\hline Alto Selva Alegre & 34 \\
Cayma & 21 \\
Cercado & 26 \\
Cerro Colorado & 55 \\
Hunter & 21 \\
José Luis Bustamante y Rivero & 27 \\
Marianao Melgar & 15 \\
Miraflores & 22 \\
Paucarpata & 55 \\
Sachaca & 8 \\
Socabaya & 28 \\
Tiabaya & 11 \\
Yanahuara & 11 \\
Total & $\mathbf{3 3 4}$ \\
\hline
\end{tabular}

Para determinar el tamaño de la muestra se utilizó una fórmula con $95 \%$ de confianza, se trata por tanto de un muestreo probabilístico. Mientras que para seleccionar a las personas se trabajó con la técnica de muestreo por racimos.

\section{Materiales}

Se ha utilizado el Inventario de Integración Familiar que estaba compuesto originalmente por 64 reactivos ubicados en una escala tipo Likert con cinco niveles de respuesta que van desde siempre, casi siempre, a veces, casi nunca y nunca; con valores de 5 a 1 . Se optó por una escala de Likert porque presenta mayores ventajas, al estar centrada en el sujeto, enfatizar sus diferencias individuales, así como por su facilidad y rapidez en el proceso de construcción y validación (Méndez y Peña, 2006).
En el apartado de resultados, se detallan los análisis estadísticos efectuados para obtener la validez y la confiabilidad de la prueba así como los respectivos baremos. Al término de estos procedimientos, se obtuvo una versión definitiva del instrumento, compuesto por 52 ítems que se presenta como anexo.

\section{Procedimientos}

Para aplicar el material en los distritos de Arequipa se contó con la participaron de cuatro colaboradores que recogieron la información según las especificaciones de la muestra. Por tanto, se preguntaba previamente si los encuestados tenían hijos y si eran jefes de familia. La aplicación de los mismos se hizo entre los meses de enero y marzo del 2013. Solo fueron encuestados jefes de familia en sus propias viviendas, y tras explicarles el objetivo del estudio se les hizo firmar el consentimiento informado y se procedió a aplicar individualmente el Inventario de Integración Familiar. El tiempo aproximado de respuesta fue de 10 a 15 minutos por persona.

\section{RESULTADOS}

En primer lugar se confeccionó un banco de preguntas que fueron sometidas a dos jueces expertos en temas de familia. En ambos casos se trató de profesionales con estudios de maestría y doctorado, que cuentan con experiencia en el tema de familia a nivel académico y profesional. Ambos jueces hicieron observaciones mínimas al instrumento, las cuales solamente completaron el sentido de cinco reactivos. Ninguna pregunta se cambió, por tanto el juicio de los expertos fue favorable, y como no presentó discrepancias, no fue necesaria la intervención de un tercer juez (Hernández, Fernández y Baptista, 2010). De modo que podemos decir que el instrumento cuenta con validez de contenido.

Para determinar la homogeneidad de contraste se aplicó el inventario a 334 personas de diversos distritos de la ciudad y se hizo una correlación ítemtest, mediante la cual se detectaron nueve ítems que no cumplieron el criterio estadístico mínimo que implica alcanzar una correlación con un coeficiente de .20 . 
Los ítems eliminados fueron los siguientes:

- $\mathrm{N}^{\circ}$ 05: "Le oculto ideas, cosas y situaciones que han ocurrido a mi pareja" ( $\mathrm{r}=-.241)$

- $\quad \mathrm{N}^{\circ}$ 09: "Le doy o le he dado motivos a mi pareja para desconfiar de mî" ( $\mathrm{r}=-.108)$

- $\mathrm{N}^{\circ}$ 20: "No contradigo lo que dice mi esposa(a) delante de mis hijos" ( $\mathrm{r}=-.100)$

- $\mathrm{N}^{\mathrm{o}}$ 40: "Mis hijos se insultan hirientemente" ( $\mathrm{r}=$ $.167)$

- $\quad N^{\circ}$ 47: "Papá (mamá) pasan mucho tiempo fuera de casa" $(\mathrm{r}=-.048)$

- $\mathrm{N}^{\circ}$ 50: "Es aburrido estar en casa y pasar tiempo con mi familia" ( $r=-.139)$

- $\mathrm{N}^{\circ}$ 54: "Las preocupaciones laborales incluyen en el clima familiar" $(\mathrm{r}=-.020)$

- $\mathrm{N}^{\circ}$ 56: "Cada uno hace su vida sin meterse en las cosas de los demás" ( $r=-.156)$

- $\mathrm{N}^{\mathrm{o}}$ 62: "Hay cosas que nunca comentaría con mi pareja por temor" $(r=.191)$

Tabla 3.

\section{Correlaciones ítem-test}

\begin{tabular}{llllllll}
\hline $\mathbf{N}^{\mathbf{0}}$ & $\mathbf{r}$ & $\mathbf{N}^{\mathbf{0}}$ & $\mathbf{r}$ & $\mathbf{N}^{\mathbf{0}}$ & $\mathbf{r}$ & $\mathbf{N}^{\mathbf{0}}$ & $\mathbf{r}$ \\
\hline $\mathbf{1}$ & .548 & $\mathbf{1 7}$ & .505 & $\mathbf{3 3}$ & .571 & $\mathbf{4 9}$ & .457 \\
$\mathbf{2}$ & .594 & $\mathbf{1 8}$ & .439 & $\mathbf{3 4}$ & .517 & $\mathbf{5 0}$ &.- \\
& & & & & & & 139 \\
$\mathbf{3}$ & .291 & $\mathbf{1 9}$ & .408 & $\mathbf{3 5}$ & .566 & $\mathbf{5 1}$ & .491 \\
$\mathbf{4}$ & .470 & $\mathbf{2 0}$ & -.100 & $\mathbf{3 6}$ & .479 & $\mathbf{5 2}$ & .521 \\
$\mathbf{5}$ & - & $\mathbf{2 1}$ & .548 & $\mathbf{3 7}$ & .568 & $\mathbf{5 3}$ & .468 \\
& .241 & & & & & & \\
$\mathbf{6}$ & .460 & $\mathbf{2 2}$ & .566 & $\mathbf{3 8}$ & .586 & $\mathbf{5 4}$ & - \\
& & & & & & & .020 \\
$\mathbf{7}$ & .555 & $\mathbf{2 3}$ & .470 & $\mathbf{3 9}$ & .476 & $\mathbf{5 5}$ & .359 \\
$\mathbf{8}$ & .511 & $\mathbf{2 4}$ & .538 & $\mathbf{4 0}$ &.- & $\mathbf{5 6}$ & - \\
& & & & & .167 & & .156 \\
$\mathbf{9}$ & - & $\mathbf{2 5}$ & .470 & $\mathbf{4 1}$ & .498 & $\mathbf{5 7}$ & .639 \\
& .108 & & & & & & \\
$\mathbf{1 0}$ & .422 & $\mathbf{2 6}$ & .609 & $\mathbf{4 2}$ & .499 & $\mathbf{5 8}$ & .564 \\
$\mathbf{1 1}$ & .545 & $\mathbf{2 7}$ & .617 & $\mathbf{4 3}$ & .571 & $\mathbf{5 9}$ & .582 \\
$\mathbf{1 2}$ & .582 & $\mathbf{2 8}$ & .591 & $\mathbf{4 4}$ & .531 & $\mathbf{6 0}$ & .566 \\
$\mathbf{1 3}$ & .569 & $\mathbf{2 9}$ & .567 & $\mathbf{4 5}$ & .577 & $\mathbf{6 1}$ & .546 \\
$\mathbf{1 4}$ & .573 & $\mathbf{3 0}$ & .561 & $\mathbf{4 6}$ & .459 & $\mathbf{6 2}$ & .191 \\
$\mathbf{1 5}$ & .607 & $\mathbf{3 1}$ & .556 & $\mathbf{4 7}$ & - & $\mathbf{6 3}$ & .517 \\
& & & & & .048 & & \\
$\mathbf{1 6}$ & .558 & $\mathbf{3 2}$ & .565 & $\mathbf{4 8}$ & .455 & $\mathbf{6 4}$ & .312 \\
\hline
\end{tabular}

Ocho de los ítems eliminados correlacionaron negativamente, pero en general no alcanzaron la puntuación mínima para formar parte de la prueba, ya que aparentemente no son representativos de la prueba. El número restante de ítems presenta correlaciones superiores a .20, de modo que se consideran representativos del instrumento, y por lo tanto cuentan con validez de criterio. En la Tabla 3 se pueden apreciar todos los valores de las correlaciones ítem-test. Aparte de los ítems con un nivel bajo de correlación o inversa, solamente el ítem 3 (Le soy fiel a mi pareja sin importar las circunstancias) obtuvo un índice bajo aunque positivo y aceptable $(r=.291)$. Una vez eliminados los ítems con correlaciones bajas o negativas, quedaron 55 reactivos que fueron sometidos a un análisis factorial para obtener la validez de constructo.

Tabla 4

Medidas de adecuación de las variables al análisis factorial

\begin{tabular}{lrl}
\hline $\begin{array}{l}\text { Medida de adecuación } \\
\text { muestral de Kaiser- } \\
\text { Meyer-Olkin }\end{array}$ & KMO & .922 \\
\hline $\begin{array}{l}\text { Prueba de } \\
\text { esfericidad } \\
\text { de Bartlet }\end{array}$ & $\begin{array}{r}\text { Chi- } \\
\text { cuadrado } \\
\text { aproximado }\end{array}$ & \\
& gl & 8685.187 \\
& Sig. & .00 \\
\hline
\end{tabular}

Se obtuvo entonces un valor alto de adecuación muestral de KMO: .922, la cual admite la factorización de la matriz de correlaciones, con el fin de realizar un análisis factorial exploratorio (Kaplan y Saccuzzo, 2006). El valor Chi-cuadrado aproximado es de 8685.187, que para 1485.00 grados de libertad tiene un nivel de significancia de .000 . Estos resultados nos permiten rechazar la hipótesis nula de $99 \%$, afirmando que nuestra matriz puede ser factorizada. 
Tabla 5

\section{Suma de las extracciones al cuadrado}

\begin{tabular}{lrcc}
\hline Factor & Total & $\begin{array}{c}\text { \% de la } \\
\text { varianza }\end{array}$ & $\begin{array}{l}\text { \% } \\
\text { acumulado }\end{array}$ \\
\hline $\mathbf{0 1}$ & 16.217 & 29.486 & 29.486 \\
$\mathbf{0 2}$ & 3.944 & 7.172 & 36.658 \\
$\mathbf{0 3}$ & 2.896 & 5.266 & 41.924 \\
$\mathbf{0 4}$ & 2.097 & 3.813 & 45.738 \\
$\mathbf{0 5}$ & 1.600 & 2.910 & 48.649 \\
$\mathbf{0 6}$ & 1.435 & 2.609 & 51.258 \\
$\mathbf{0 7}$ & 1.383 & 2.514 & 53.773 \\
$\mathbf{0 8}$ & 1.295 & 2.354 & 56.127 \\
$\mathbf{0 9}$ & 1.227 & 2.231 & 58.359 \\
$\mathbf{1 0}$ & 1.119 & 2.035 & 60.394 \\
$\mathbf{1 1}$ & 1.079 & 1.963 & 62.358 \\
$\mathbf{1 2}$ & 1.002 & 1.822 & 64.180 \\
\hline
\end{tabular}

Al hacer la reducción de factores con rotación varimax se obtuvo una distribución de 12 factores, donde el primer factor explicaba el $29,486 \%$ de la varianza total de la prueba (ver Tabla 5). Este solo hecho podría ser un indicador de que el Inventario de Integración Familiar es un instrumento unidimensional (Burga, 2006), dado que además el valor del porcentaje que explica la varianza total de los otros ítems era bajo, como se puede apreciar en la Tabla 5.

Debido a esto, es que se eliminaron los ítems con cargas factoriales menores .30, lo que dio por resultado que casi todos los ítems se ubicaran en el primer factor, de modo que solo tres se ubicaban en otros factores. Las cargas factoriales se aprecian en la Tabla 6.

Esto determinó la presencia de cuatro factores, de modo que el primer factor agrupa la mayoría de 55 ítems y solo tres se encuentran distribuidos en tres factores diferentes. Estos cuatro factores, constituyen entonces, los componentes básicos de la escala, pero dada la distribución de las cargas factoriales y el peso porcentual de la varianza en el primer factor, se optó por eliminar los tres ítems que no se ubicaron en el mismo.

Los ítems eliminados fueron:
- $\quad \mathrm{N}^{\circ}$ 03: "Le soy fiel a mi pareja sin importar las circunstancias" $(\mathrm{r}=.461)$

- $\quad \mathrm{N}^{\circ} 20$ : "Enseño a mis hijos a respetar a los demás y a ser honestos" ( $\mathrm{r}=.447)$

- $\mathrm{N}^{\mathrm{o}}$ 55: "Los límites están bien claros entre los miembros de mi familia" $(\mathrm{r}=.585)$

De este modo, el Inventario de Integración Familiar quedó como un instrumento unidimensional que está compuesto por un solo factor. Por tanto como producto del análisis factorial en dos etapas se obtuvo por resultado un instrumento unidimensional. Para el cálculo de la confiabilidad, se obtuvo un valor Alfa de Cronbach de $\alpha=.739$, por lo tanto el inventario resultante es confiable, considerando que el valor alfa encontrado es mayor de .70 (Kaplan y Saccuzzo, 2006).

Tabla 6.

Matriz de componentes con rotación varimax

\begin{tabular}{|c|c|c|c|c|}
\hline Ítems & Factor 1 & Factor 2 & Factor 3 & Factor 4 \\
\hline 01 & .628 & & & \\
\hline 02 & .664 & & & \\
\hline 03 & & & .461 & \\
\hline 04 & .579 & & & \\
\hline 05 & .516 & & & \\
\hline 06 & .565 & & & \\
\hline 07 & .567 & & & \\
\hline 08 & .470 & & & \\
\hline 09 & .627 & & & \\
\hline 10 & .680 & & & \\
\hline 11 & .619 & & & \\
\hline 12 & .561 & & & \\
\hline 13 & .637 & & & \\
\hline 14 & .634 & & & \\
\hline 15 & .455 & & & \\
\hline 16 & .391 & & & \\
\hline 17 & .426 & & & \\
\hline 18 & .554 & & & \\
\hline 19 & .539 & & & \\
\hline 20 & & .447 & & \\
\hline 21 & .462 & & & \\
\hline 22 & .494 & & & \\
\hline 23 & .602 & & & \\
\hline 24 & .584 & & & \\
\hline 25 & .567 & & & \\
\hline 26 & .560 & & & \\
\hline 27 & .524 & & & \\
\hline
\end{tabular}




\begin{tabular}{|c|c|c|c|c|}
\hline $\begin{array}{l}\text { Ítems } \\
28\end{array}$ & $\begin{array}{l}\text { Factor } 1 \\
.586\end{array}$ & Factor 2 & Factor 3 & Factor 4 \\
\hline 29 & .560 & & & \\
\hline 30 & .519 & & & \\
\hline 31 & .435 & & & \\
\hline 32 & .534 & & & \\
\hline 33 & .407 & & & \\
\hline 34 & .530 & & & \\
\hline 35 & .565 & & & \\
\hline 36 & .562 & & & \\
\hline 37 & .524 & & & \\
\hline 38 & .496 & & & \\
\hline 39 & .522 & & & \\
\hline 40 & .574 & & & \\
\hline 41 & .588 & & & \\
\hline 42 & .507 & & & \\
\hline 43 & .427 & & & \\
\hline 44 & .602 & & & \\
\hline 45 & 561 & & & \\
\hline 46 & .602 & & & \\
\hline 47 & .586 & & & \\
\hline 48 & .415 & & & \\
\hline 49 & .615 & & & \\
\hline 50 & .596 & & & \\
\hline 51 & .541 & & & \\
\hline 52 & .601 & & & \\
\hline 53 & .579 & & & \\
\hline 54 & .506 & & & \\
\hline 55 & & & & .585 \\
\hline
\end{tabular}

Asimismo, se establecieron los baremos con los respectivos percentiles para los valores obtenidos. Estos baremos generaron tres rangos de respuesta para la integración familiar.

- Un nivel bajo de integración familiar se ubica entre valores de 94 a 200.

- Un valor moderado de integración familiar se ubica en un rango de 204 a 235.

- Un nivel alto de integración familiar toma puntajes desde 237 a 260.

Los valores del baremo con sus respectivos percentiles y valores descriptivos se aprecian en la Tabla 7.
Tabla 7

Baremos del Inventario de Integración Familiar

\begin{tabular}{|c|c|c|}
\hline Nivel & Percentiles & $\begin{array}{c}\text { Puntuación } \\
\text { Directa }\end{array}$ \\
\hline \multirow{6}{*}{ Bajo } & 1 & 94 \\
\hline & 5 & 146 \\
\hline & 10 & 170 \\
\hline & 15 & 180 \\
\hline & 20 & 192 \\
\hline & 25 & 200 \\
\hline \multirow{9}{*}{ Medio } & 30 & 204 \\
\hline & 35 & 209 \\
\hline & 40 & 212 \\
\hline & 45 & 217 \\
\hline & 50 & 220 \\
\hline & 55 & 226 \\
\hline & 60 & 229 \\
\hline & 65 & 232 \\
\hline & 70 & 235 \\
\hline \multirow{9}{*}{ Alto } & 75 & 237 \\
\hline & 80 & 240 \\
\hline & 85 & 242 \\
\hline & 90 & 252 \\
\hline & 95 & 260 \\
\hline & Media & 213,080 \\
\hline & DE & 33,451 \\
\hline & Mínimo & 52 \\
\hline & Máximo & 260 \\
\hline
\end{tabular}

Después de todo este procedimiento, se obtuvo un instrumento unidimensional de 52 ítems dispuestos en una escala de tipo Likert con cinco alternativas de respuesta, cuyos índices de validez y confiabilidad son aceptables.

\section{DISCUSIÓN}

Nos planteamos por objetivo la construcción de un instrumento que mida la integración familiar. Para ello seguirnos la teoría de Análisis Factorial de los Ítems (AFI), que si bien, junto con la Teoría Clásica de los Tests (TCT) constituyen modelos psicométricos lineales, en contraposición con las Teorías de Respuesta al Ítem (TRI) que se basan en modelos con distinto 
número de parámetros para cada ítem; no se aprecian diferencias sustantivas entre los modelos AFI y TRI, al momento de evaluar la validez estructural. Por tanto se concluye que existe equivalencia entre los modelos AFI y TRI, a pesar de que en el AFI los datos son tratados como continuos y en el TRI son tratados como categorías de respuesta (Denegri, 2008).

Basándonos en el modelo AFI, hemos hallado la validez del inventario, entendiendo por esta, "la concordancia entre la puntuación o medida de una prueba y la cualidad que se cree que está midiendo" (Kaplan y Saccuzzo, 2006, p. 134). De ahí que como dice Denegri (2008), la validez se refiere a la necesidad de garantizar que las inferencias derivadas del instrumento de medida sean pertinentes y acordes al atributo que se supone que está evaluando. En ese sentido, al hacer la correlación ítem-test, se eliminaron nueve reactivos, de los cuáles ocho tenían correlaciones negativas, a excepción del ítem 62 (Hay cosas que nunca comentaría con mi pareja por temor) que aunque correlaciona positivamente, no fue superior a .20. El resto de ítems mostró correlaciones superiores a .30, a excepción del ítem 3 , lo que sugiere que cada reactivo representa la variable estudiada, es decir que cada ítem es una muestra de la variable que se mide (Kaplan y Saccuzzo, 2006).

También se realizó un análisis factorial. Esta técnica fue utilizada por primera vez en 1904 por Charles Spearman con el fin de determinar la estructura de las covarianzas entre las variables - para nuestro caso ítems- y bautizar los factores encontrados (Denegri, 2008). Pero después Thurstone incorporó al análisis factorial los métodos de algebra de matrices, lo cual derivó en las rotaciones. Con la rotación se pretende hallar la estructura de cada factor. Un factor es una dimensión común de datos. De acuerdo con un enfoque analítico de ítems, no se puede asignar un ítem a más de un factor (Fleming y Merino, 2005). Por tanto el valor del análisis factorial radica en el grado en que es capaz de descubrir un grupo de factores causativos que expliquen un orden psicológico subyacente en las pruebas psicológicas. Es decir que el análisis factorial tiene por objeto descubrir las dimensiones de variabilidad común, existentes en un cierto campo de fenómenos (Yela, 1957).
Ahora bien, en un primer momento, se ha determinado la existencia de doce factores, sin embargo, esta primera interpretación no fue definitiva, ya que en tanto los ítems se hallaban distribuidos predominantemente en el factor 1 y la varianza explicada por el primer factor fue del $29 \%$, la prueba evidenciaba una tendencia a la unidimensionalidad. En ese sentido, un instrumento será unidimensional si el primer factor explica por lo menos el $40 \%$ de la varianza, aunque también se menciona que basta con valores superiores a $30 \%$, otros sugieren el $20 \%$ y otros aún, el 17\% (Burga, 2006). Es importante entonces, que el material muestre un solo factor dominante, como ha ocurrido con el Inventario de Integración Familiar, y por lo tanto lo constituye de manera unidimensional. Esta unidimensionalidad implica que un solo constructo se encuentra en la base de todos los ítems. Sin embargo, como en una segunda factorización, se obtuvo cuatro factores distribuidos desigualmente, se optó por eliminar los ítems de los factores con menos cargas factoriales, para conseguir la unidimensionalidad de nuestro instrumento, ya que como señalan Kaplan y Saccuzzo (2006) es preferible que se omitan los reactivos que no tienen carga en el factor dominante.

Si bien en la práctica ningún instrumento es perfectamente unidimensional, se espera tener pruebas que muestren unidimensionalidad. Ello no implica empero, que las respuestas se deban a un único proceso psicológico (Burga, 2006). Para el caso de la integración familiar, se trata de un constructo complejo que comprende la salud y la satisfacción de necesidades trascendentes que devienen en estados de bienestar, y que implican el respeto, el diálogo y la comunión entre los miembros de la familia en función de su ciclo de vida. Se trata por tanto de un constructo de alta complejidad.

$\mathrm{Al}$ respecto, se ha planteado que las pruebas con constructos complejos son menos confiables (Kaplan y Saccuzzo, 2006). El índice de confiabilidad obtenido para nuestro instrumento fue de $\alpha=0.739$ mediante el índice de consistencia interna Alpha de Cronbach, con una cantidad final de 52 ítems. En ese sentido, se han tenido que eliminar 12 ítems del banco de reactivos inicialmente planteados, lo que podría restar confiabilidad al inventario (Magnusson, 1969), 
pero como la integración familiar es un constructo complejo, no es necesario buscar una confiabilidad superior de .90 , ya que las pruebas son más confiables si son unidimensionales.

En resumen, dado que la integración familiar es un constructo complejo y el instrumento exhibe una confiabilidad superior a .70 y las correlaciones ítemtest han sido superiores a .30 , podemos concluir que el Inventario de Integración Familiar es un material unidimensional tanto válido como confiable. Es importante eso sí, tener en cuenta antes de su aplicación, que es aplicable a padres de familia, es decir que no basta estar casado o convivir, sino también tener hijos, por el tipo de reactivos que componen el inventario. En ese sentido, el Inventario de Integración Familiar cuenta con ciertas limitaciones, ya que no es aplicable a familias sin hijos, u hogares monoparentales, ya que los ítems abarcan cuestiones propias del holón conyugal, parental, fraternal y familiar.

Se espera que con los baremos ofrecidos, se pueda valorar la integración familiar de las familias peruanas y profundizar en el conocimiento de este constructo a través de futuras investigaciones. De este modo, esperamos que este instrumento y sus múltiples aplicaciones, contribuyan a la formulación de una cultura familiar más armónica, sólida y saludable (Melina, 2010).

\section{REFERENCIAS}

Arellano, R. (2012). Somos más que siesta y fiesta. Doce mitos y verdades sobre América Latina. Lima: Planeta.

Arias, E. (2011). Algunas consideraciones sobre la familia y la crianza desde un enfoque sistémico. Revista de Psicología de Arequipa, 2(1), 32-46.

Arias, E. (2013). Agresión y violencia en la adolescencia: La importancia de la familia. Avances en Psicología, 21(1), 23-34.

Baessa de, Y. \& Fernández, F. J. (2003). Factores del hogar en el rendimiento escolar. Revista de Psicología de la PUCP, 21(2), 311-331.
Burga, A. (2006). La unidimensionalidad de un instrumento de medición: perspectiva factorial. Revista de Psicología de la PUCP, 24(1), 53-80.

Caffarra, C. (2011). La familia: Un lugar de la experiencia de comunión. Persona y Cultura. 9(9), 69-79.

Covarrubias, M. A. y Gómez, J. (2012). Transformación y contemporaneidad de la familia: el caso de las familias mexicanas. Revista de Psicología de la $U C V, 14(2), 244-259$.

Denegri, U. K. (2008). Equivalencia entre los modelos de análisis factorial de los ítems y teoría de respuesta a los ítems en la evaluación de las propiedades psicométricas de los instrumentos de medición psicológica. Revista Peruana de Psicometría, 1(1), Disponible en: http://www. rppsicometria.com.pe/articulos/vol1.art.3.pdf

Eguiluz, L. L.; Calvo, R. M. y De la Orta, D. (2012). Relación entre la percepción de la satisfacción marital, sexual y la comunicación en parejas. Revista de Investigaciones Psicológicas, 1(1), 1528.

Fleming, J. S. y Merino, C. (2005). Medidas de simplicidad y de ajuste factorial: un enfoque para la evaluación de escalas construidas factorialmente. Revista de Psicología de la PUCP, 23(2), 249266.

Guerra, R. (2004). ¿Familia o familias? Familia natural y funcionalidad social. Persona y Cultura. 3(3), $87-103$.

Haley, J. (2002). Terapia para resolver problemas. Nuevas estrategias para una terapia familiar eficaz. Buenos Aires: Amorrotu Editores.

Hellinger, B. (2002). Lograr el amor en la pareja. Barcelona: Herder.

Hellinger, B. (2003). Reconocer lo que es. Barcelona: Herder.

Hellinger, B. (2005). Órdenes de amor. Barcelona: Herder.

Hernández, R.; Fernández, C. y Baptista, P. (2011). Metodología de la investigación. México: McGraw-Hill. 
Instituto Nacional de Estadística e Informática. (2013). Perú, evolución de los indicadores de los objetivos de desarrollo del Milenio al 2011. Lima. Centro de Ediciones del INEI.

Jadue, G. (1996). Características familiares de los hogares pobres que contribuyen al bajo rendimiento o al fracaso escolar de los niños. Revista de Psicología de la PUCP, 14(1), 35-45.

Jadue, G. (2000). Algunas características familiares y de la escuela que contribuyen a la etiología de la tensión emocional. Revista de Psicología de la PUCP, 18(1), 69-85.

Juan Pablo II. (1981). Familiaris Consortio. Lima: Epiconsa.

Kaplan, R. M. \& Saccuzzo, D. P. (2006). Pruebas psicológicas. Principios, aplicaciones y temas. México: Thomson.

Laguna, J. P. \& Rodríguez, A. S. (2008). Comportamientos socioemocionales de resiliencia en preescolares procedentes de hogares mono y biparentales. Revista de Psicología de la UCSM, $5,52-65$.

Magnusson, D. (1969). Teoría de los tests. México: Trillas.

Mayorga, E. y Ñiquen, M. (2010). Satisfacción familiar y expresión de cólera-hostilidad en adolescentes escolares que presentan conductas antisociales. Revista de Investigaciones Psicológicas, 1(1), 8792.

Melina, L. (2010). Por una cultura de la familia. El lenguaje del amor. Lima: Universidad Católica San Pablo.

Méndez, L. M. \& Peña, J. A. (2006). Manual práctico para el diseño de la escala Likert. México: Trillas.

Merino, C.; Díaz, M. y DeRoma, V. (2004). Validación del inventario de conductas parentales: un análisis factorial confirmatorio. Persona, 7, 145-162.

Minuchin, S. \& Fishman, H. (1996). Técnicas de terapia familiar. México: Paidós.

Oporto, C. \& Zanabria, L. (2006). Inteligencia emocional en hijos de familias nucleares $y$ monoparentales. Revista de Psicología de la UCSM, 3, 25-36.

Perriaux, J. (2011). La familia ante algunos desafíos de la realidad actual. Persona y Cultura. 9(9), 12-33.

Pinzas, J. (1987). Notas para una propuesta sobre las relaciones entre familia y educación. Rol de los padres en el aprendizaje de la lectura y la comprensión lectora. Revista de Psicología de la PUCP, 5(2), 177-188.

Portilla, Ch.; Salinas, P. \& Bueno, K. (2006). Apego en infantes institucionalizados y no institucionalizados. Revista de Psicología de la UCSM, 3, 5-24.

Portilla, Ch.; Romero, B. \& Gonzales, J. (2008). Apego infantil en madres que trabajan y no trabajan. Revista de Psicología de la UCSM, 5, 5-21.

Prado, R. y Del Águila, M. (2004). Estructura $\mathrm{y}$ funcionamiento familiar en adolescentes resilientes. Teoría e Investigación en Psicología, 13, 85-113.

Prado, T. R. y Del Águila, M. (2010). Ajuste y satisfacción en parejas que trabajan. Revista de Investigaciones Psicológicas, 1(1), 38-52.

Rebaza, R. P. y Julca, M. B. (2009). Satisfacción marital $\mathrm{y}$ ansiedad por concebir un hijo en mujeres con diagnóstico de infertilidad. Revista de Psicología de la UCV, 11, 79-96.

Ríos, J. A. (2003). Los ciclos vitales en la familia y la pareja. ¿Crisis u oportunidades? Madrid: Editorial CCS.

Rodríguez, J. M. (2006). Amor conyugal. Serie Familia Hoy. Arequipa: Universidad Católica San Pablo.

Rodríguez, J. M. (2008). Vida espiritual en el matrimonio. Serie Familia Hoy. Arequipa: Universidad Católica San Pablo.

Salvatierra, B. (1992). ¿Qué hago? Arequipa: UNSA.

Salvatierra, B. (1993). El matrimonio. Arequipa: UNSA.

Salvatierra, B. (1997). Orientación psicológica para la familia. Arequipa: UNSA. 
Scola, A. (2001). Hombre-mujer. El misterio nupcial. Arequipa: Universidad Católica San Pablo.

Serretti, M. (2011). Naturaleza de la comunión. Ensayo sobre la relación. Arequipa: Universidad Católica San Pablo.

Silva, C. \& Argote, C. (2007). Actitudes hacia matrimonio y divorcio en jóvenes procedentes de familias intactas y divididas. Revista de Psicología de la UCSM, 4, 29-37.

Sobrino, L. (2008). Niveles de satisfacción familiar y de comunicación entre padres e hijos. Avances en Psicología, 16(1), 109-137.
Tirado, P.; Álvarez, V.; Chávez, M.; Holguín, S.; Honorio, A.; Moreno, M.; Sánchez, N.; Shimajuko, A. y Uribe, M. (2008). Satisfacción familiar y salud mental en alumnos universitarios ingresantes. Revista de Psicología de la UCV, 10, 42-48.

Yaya, M. A.; Vilches, F. \& Portilla, Ch. (2009). Apego en adolescentes, nivel socioeconómico y género. Revista de Psicología de la UCSM, 6, 40-57.

Yela, M. (1957). La técnica del análisis factorial. Un método de investigación en psicología y pedagogía. Madrid: Biblioteca Nueva.

Fecha de recepción: 21 de agosto de 2013

Fecha de aceptación: 25 de septiembre de 2013 


\section{Anexos \\ Reactivos del Inventario de Integración familiar}

1. Estoy satisfecho(a) con mi matrimonio.

2. Converso con mi pareja sobre las cosas que pasan día a día.

3. Comparto hobbies y pasatiempos con mi pareja.

4. Salgo con mi pareja a diversos eventos que tienen lugar fuera de casa.

5. Converso constantemente con mi pareja sobre nuestra relación.

6. $\quad$ Mi pareja comprende como pienso y como me siento.

7. Me encuentro satisfecho(a) en mi vida íntima con mi pareja.

8. $\quad$ En el matrimonio me veo realizado(a).

9. Con mi pareja valoramos las mismas cosas.

10. Tengo tiempo destinado especialmente para estar con mi pareja.

11. Admiro a mi pareja.

12. Saludo y me despido afectuosamente de mi esposa(o) cuando salgo o llego a casa.

13. Sorprendo a mi esposo(a) con algo que le agrada.

14. Mi pareja y yo estamos pendientes de las necesidades de nuestros hijos.

15. Conozco a los profesores de mis hijos.

16. Mis hijos acuden a mí o a su madre (padre) cuando tienen algún problema.

17. Mis hijos suelen obedecer las órdenes que les damos en casa.

18. Considero que mis hijos me tienen confianza.

19. La opinión de mis hijos es tomada en cuenta.

20. Conozco a la familia de los amigos de mis hijos.

21. Mantenemos con firmeza las órdenes y acuerdos que les damos a nuestros hijos.

22. En casa asignamos responsabilidades domésticas a nuestros hijos.

23. Mi esposa(a) y yo les damos un buen ejemplo a nuestros hijos.

24. Respetamos las decisiones que toman nuestros hijos.

25. Ayudo a mis hijos a realizar sus deberes escolares.

26. Conversamos constantemente sobre los temas que les preocupa a mis hijos.

27. Conozco a los amigos de mis hijos

28. Sé cuáles son los hobbies e intereses de mis hijos.

29. Inculcamos valores a nuestros hijos.

30. Mis hijos comparten tiempo y juegos.

31. Ellos se cuidan entre sí.

32. Se preocupan por el bienestar del otro.

33. Colaboran entre sí para cumplir con los quehaceres de la casa.

34. Realizan algún deporte o actividad artística juntos.

35. Conversan sobre las cosas de la casa, la escuela o el trabajo.

36. Entre mis hijos hay confianza.

37. Se conocen mutuamente.

38. Todos estamos satisfechos en nuestra familia.

39. En mi familia las decisiones importantes las tomamos todos juntos.

40. Es costumbre almorzar todos juntos.

41. En mi familia participamos con regularidad de alguna actividad religiosa.

42. Estoy satisfecho(a) con el ambiente familiar de mi familia.

43. Conversamos sobre lo que hemos visto en la TV o en las películas.

44. Acostumbramos salir de paseos o hacer viajes familiares.

45. En mi familia acostumbramos ver películas todos juntos.

46. Mis hijos tienen una relación cercana con sus abuelos.

47. Todos colaboramos con la limpieza y el orden de la casa.

48. Estoy satisfecho(a) con la relación que tengo con mis suegros y cuñados.

49. Cada miembro de mi familia tiene sus propias responsabilidades.

50. Considero que hay momentos que son solo de mi pareja.

51. Las decisiones, los gustos e intereses de cada uno se respetan.

52. Mi pareja tiene proyectos en la vida que le gustaría hacer realidad. 